\title{
Interactive Watershed Optimization in the Presence of Spatially-varying and Uncertain Stakeholder Preferences
}

\author{
Meghna Babbar Sebens \\ School of Civil and Construction Engineering \\ Oregon State University \\ Corvallis, USA, meghna@oregonstate.edu
}

\author{
Kenneth R. Cannady Shultz \\ School of Civil and Construction Engineering \\ Oregon State University \\ Corvallis,USA
}

\author{
Snehasis Mukhopadhyay \\ Department of Computer and Information \\ Science \\ IUPUI, Indianapolis \\ USA, smukhopa@iupui.edu
}

\begin{abstract}
Watershed planning over a geographic area is a difficult task primarily due to the presence of large number of stakeholders and decision makers whose intrinsic conflicting and/or subjective preferences often lead to uncertainty in perceived fitness of planning decisions. Deciding which watershed strategy should be implemented at what location requires a participatory approach to design and decision making, if adoption of landscape decisions is critical to success. Analytical participatory design (APD) approaches aim to enable farmers, environmentalists, government agencies, and other stakeholders to visualize the landscape, explore and design competitive scenarios of implementing certain management practices on the landscape. Since these approaches improve decision makers' awareness of opportunities and constraints in the co existing physical and human systems, it is hypothesized that they can be used to generate acceptable decisions that are robust to uncertainties in stakeholder preferences.
\end{abstract}

An APD method based on Interactive Optimization is described in this paper and tested for design of wetlands in a study watershed site (Eagle Creek Watershed) in the state of Indiana. The method is then used to test research hypothesis by involving multiple virtual stakeholders as surrogates to diverse human users and their preferences. The results indicate that, while, as expected, the interactive optimization approach results in lower values of the financial and environmental objective criteria (which are being traded off against users' diverse subjective personal criteria), it also results in a relatively high degree of user consensus, indicating high likelihood of adoption of the generated solutions by the stakeholders.

Keywords watershed planning, interactive optimization, genetic algorithms, consensus

\section{INTRODUCTION}

Water is an importance resource and necessity for the sustenance of human civilization. So much so that many scholars predict that water will be to the 21 st century what oil was to the 20th [1]. Water management, therefore, poses one of the biggest scientific challenges facing humanity today. Too much water can lead to flood, too little can lead to drought, and poor water quality can lead to disastrous public health and ecological health consequences. Management of watershed systems and water resources requires a rigorous understanding of not only the physical and biological laws that govern how water is stored, regulated, and routed in the natural and built environment, but also processes related to watershed stakeholders (i.e. landowners, consultants, managers, and agency personnel, etc.) that affect how decisions for water management are created and adopted. And while significant research has been conducted over multiple decades to understand how the uncertainty in data, simulation models, and modelers influence the way we predict the behavior of water in our physical environments, research on the estimation of uncertainty in human (stakeholder) related processes and human dimensions data has been relatively scarce.

It is generally agreed that in any type of natural resources management problem (e.g., water management, landscape management, etc.) better decisions will be implemented with less conflict if the decision making is driven by the social network of multiple stakeholders who are affected by these decisions [2]. As a result, research on analytical participatory modeling and design methods, which directly engage with humans to better characterize data on stakeholders' diverse opinions, preferences, and biases when decision alternatives are being generated, have become considerably popular in the recent past [3]. The answer to the question "How does one measure and characterize uncertainty in preferences of stakeholders in participatory modeling and participatory design methods?" has, hence, become critical in advancing the science of participatory, natural resource management. The overarching goal of this research paper is to develop and test a novel, mathematical and computational approach based on user consensus for characterizing and coping with uncertainty in diverse stakeholders' willingness to adopt proposed watershed conservation decisions. Specifically, the following are the specific objectives of this paper:

Objective 1: Investigate how users' preferences for watershed conservation decisions (specifically, wetland decisions in this paper) can be represented via preference models, and then, based on the uncertainty in their willingness to participate in conservation planning, can be combined into a community consensus metric for evaluating acceptability of proposed design alternatives.

Objective 2: Evaluate the effectiveness of using community consensus metric in driving the search process of an Interactive Multi-objective Optimization algorithm in order to generate design alternatives that are robust to different degrees of stakeholder participation in a test watershed the Eagle Creek Watershed in the state of Indiana, USA.

This is the author's manuscript of the article published in final edited form as: 


\section{CURRENT STATE OF KNOWLEDGE}

Interactive Optimization: In a 1985 seminal paper, Fisher [4] motivated a discussion on optimization/search algorithms that were interactive and allowed humans to be a part of the search process, especially for problems where human thought processes would provide advantage to the "algorithmic thinking" employed by a computer for example, processes related to visual perception, strategic thinking, and the ability to learn. According to his discussions, incorporating human interaction within the optimization algorithms could (a) facilitate model specification and revisions, (b) help cope with problem aspects that are difficult to quantify, and (c) assist in the learning and problem-solving process. Also known as human-guided search [5], such human-in-the-loop type of interactive search/optimization techniques have demonstrated promising results in applications such as space shuttle scheduling [6], vehicle routing [7], face image generation [8], constraint-based graph drawing [9], and more recently by the authors and others for environmental problems [10] [14]. Human-centered interactive optimization approaches are different from, and far more superior than, the interactive optimization approaches previously proposed by multiple researchers, such as [15], where the decision makers were asked to provide feedback on alternatives only on the basis of the quantitative objective functions. In human-centered interactive optimization algorithms, the decision makers are also asked to consider the quality of decision variable values and provide feedback on the qualitative and unquantifiable aspects of the alternatives that are extremely critical to success of an optimization effort.

Interactive Watershed Optimization: Two of the authors, Babbar-Sebens and Mukhopadhyay, have developed an innovative, participatory optimization-based design approach and cyberinfrastructure for watersheds, called WRESTORE (Watershed REstoration using Spatio-Temporal Optimization of REsources) (project website: http://wrestore.iupui.edu). The human-centered interactive optimization approach in webbased WRESTORE software is based on the Interactive Genetic Algorithm with Mixed Initiative Interaction (IGAMII) algorithm proposed originally by [12]. In summary, the interaction with human users/stakeholders occurs in an iterative manner via the graphical user interfaces (GUI). In every iteration, which is called an interaction session, both the decision space of the alternatives (via maps) and the objective space of the alternatives (via graphs) are displayed to the user. The user evaluates multiple alternatives based on not only the quantitative objectives (i.e. mathematical dynamic functions of cost-benefit goals) but also based on the user's local subjective criteria or qualitative knowledge not represented in the problem formulation. Once the user has evaluated the alternatives, she/he can provide their feedback on the quality of the alternative to the IGAMII algorithm via a user rating or human rank determined on a Likert scale. The IGAMII algorithm collects different types of explicit (i.e., user ratings) and implicit (i.e., mouse clicks on the interface) feedback data, and uses the feedback to identify new alternatives that are similar to or better than the alternatives liked by the user. The underlying optimization algorithm is critical to enabling search of new alternatives, and though the IGAMII uses a multi-objective Genetic Algorithm, WRESTORE is not restricted by the type of multi-objective optimization technique and allows the user to also select from a variety of other search approaches. Over the past few years, the authors, Babbar-Sebens and Mukhopadhyay, have been investigating the effects of (a) dynamic (non stationary) user-learning [16], (b) dynamically fitted user mental models (or, surrogate mental models) [17], and (c) variability and trends in user-feedbacks, on the interactive optimization algorithm's search space [14]. But since participatory design generally involve large communities, research is needed on formal methods that combine the uncertainty arising from varied learning and perceptions in multiple users with the uncertainty that arises from imperfect knowledge and models of the physical watershed systems. Improved understanding and methods for managing these different types of uncertainty during participatory design are vital to the success of human-centered optimization approaches.

Human-Centered Interactive Optimization Methods Using Multiple User Models: The use of multiple user models essentially leads to a multi-criteria decision-making problem, where each model can be viewed as contributing a separate criterion function. This implication of multiple models for decision-making is a new research thrust in natural resources management, where the research on using multiple models (or ensemble of models) has almost entirely focused on prediction problems aiming only to improve the prediction accuracy, rather than improved decision-making. Broadly speaking, there are three approaches to decision-making, when there are multiple criteria present [18]:

(i) Combining the multiple criteria in a linear or nonlinear way into a single criteria

(ii) Determining a Pareto-optimal solution for all the criteria

(iii) Determining a "satisfying goal" for each criterion and deriving a solution for the same

In this paper, we will focus on approach (ii), where criteria are user-determined and not necessarily explicit.

Determining Pareto Optimal Solutions: Pareto Optimal: Given a set of criteria $h_{i}(x), i=1, \ldots, n$, and $x \in X$, a point $x^{*} \in X$ is Pareto optimal if and only if there does not exist another point $x \in X$, such that $h_{i}(x) \leq h_{i}\left(x^{*}\right)$ for each $i=1, \ldots, n$, and $h_{j}(x)<h_{j}\left(x^{*}\right)$ for at least one criterion $h_{j}($.$) . We have used$ multi-criteria genetic algorithms extensively to compute such Pareto-optimal solutions in our WRESTORE web-based, interactive, watershed design tool. Genetic Algorithms (GAs) have gained popularity among many practitioners who deal with discrete, non-convex, and discontinuous optimization problems. These are heuristic search algorithms that were first proposed by John Holland [19] and work with a population of possible designs. The designs are evolved using a process analogous to that of the theory of evolution. Decision variables can be encoded in any numeral base system, though binary 
coding is most popular. All possible alphabets for a particular coding are also called "alleles". These coded variables or alleles are grouped together into representative strings called "chromosomes," each of which represents a candidate design and represents the quality of the design through its "fitness". All positions in the chromosome that the alleles occupy are known as a "locus". Based on the idea of "natural selection," better designs are created by using various "genetic" GA operators (i.e. selection, crossover and mutation) on the chromosomes. The genetic operators identify, select and mix high performance building blocks to create robust designs from available chromosomes.

\section{METHODOLOGY}

In this section, we first describe the watershed study area where this research was focused on, including the watershed models used to assess impacts of wetland decisions on flooding impacts, in Section 3.1. In Section 3.2, we describe the models used to simulate community preferences of multiple stakeholders in a watershed, who may have different and many times conflicting priorities and biases towards wetlands as conservation decisions. Finally, in Section 3.3 we describe the metric for assessing community consensus and IGA approach and optimization problem formulation that was used to test the influence of stakeholder preferences and consensus on design of watershed plans involving conservation practices.

\subsection{Study Area}

Eagle Creek watershed (ECW) is located northwest of Indianapolis, and features agricultural, urban, and undeveloped land uses. This watershed has a drainage area of approximately $419 \mathrm{~km}^{2}$ and is part of the larger Upper White River Watershed. Eagle Creek drains to Eagle Creek reservoir, which is a major source of drinking water and recreation for Indianapolis and the surrounding areas. Over $60 \%$ of the watershed is agricultural, with the dominant crop being corn. Babbar-Sebens et al. [20] in their previous work identified 2953 possible sites for wetlands in this watershed on agricultural land, using a spatial analysis technique based on land topography, soils, and land use datasets. In recent years, upstream agricultural areas have released pesticides, sediments, and fertilizers into nearby streams, resulting in the Eagle Creek reservoir becoming impaired. Generally, the climate in Indiana is considered continental, with typical cumulative annual precipitation between 965 and 1,016 $\mathrm{mm}$ and average annual temperature of approximately $11^{\circ} \mathrm{C}$.

The surrogate users utilized in this research represent typical agrarian stakeholders, similar to those found in ECW, who bear two main concerns: environmental and financial. Reimer et al [21] identified these two concerns as primary motivators that farmers consider when implementing management practices, such as wetlands. In addition to these two primary motivators, their study also found that farmers consider on-farm and offfarm benefits when making their conservation decision. Typically, individuals who self-identify as stewards of the land will concern themselves with both on and off farm benefits, while individuals driven by financial constraints will focus more on direct benefits to their own property. In the absence of stakeholders available for participation in this study, we created models of agrarian stakeholders based on Reimer et al [21] study. The simulated stakeholders represented varying interests of stakeholders in environmental and financial benefits attained within their assigned sub-catchment (i.e., on-farm benefit) versus the larger watershed community (i.e., off-farm benefits).

\subsection{Simulated Stakeholders}

\subsubsection{Proposed Scoring Equation for Simulating Diverse Stakeholder Preferences for Conservation Decisions}

To model stakeholders with two cost-benefit motivators (wetland area that represents financial costs, and peak flow reduction that represents flooding benefits), this study utilized a simple deterministic scoring equation (Equation 1).

$$
\begin{gathered}
\text { Score }_{i j}=w\left(\gamma \frac{P_{F R_{i j}}}{\text { IdealPFR }_{j}}+(1-\gamma) \frac{\text { IdealArea }_{j}}{\text { Area }_{i j}}\right)+(1- \\
w)\left(\gamma \frac{\text { PFR }_{i t}}{\text { IdealPFR }_{t}}+(1-\gamma) \frac{\text { IdealArea }_{t}}{\text { Area }_{i t}}\right)
\end{gathered}
$$

Score $_{i j}$ is calculated for every stakeholder $j$ for all $i$ design alternatives, where each $j^{\text {th }}$ stakeholder is assumed to be associated with sub-catchment (or, sub-basin) $j$. The calculated score is a number between 0 and 1 and represents a stakeholder's relative preference for $i^{\text {th }}$ design alternative, with a higher score indicating a higher preference for $i^{\text {th }}$ design. The stakeholder score is determined as a weighted average of peak flow reduction $(P F R)$ and wetland area (Area) objective function values for a multi-objective wetland design optimization problem involving goals that maximize PFR and minimize Area (see [20]) for details on how objective function values of Area and PFR are calculated). A subscript $j$ is for assessing $P F R$ or Area objective function value within the specific sub-basin $j$ that the stakeholder $j$ resides in, while a subscript of $t$ is for $P F R$ or Area value for the entire watershed. In ECW there are a total of 108 sub-basins where wetlands can be implemented. The word "Ideal" preceding PFR or Area defines the ideal objective function values corresponding to the best-case scenario with respect to that variable. For PFR, the "Ideal" fitness function value corresponds to the scenario that yields the greatest reduction in peak flows, which is the scenario when all possible 2953 new wetlands are constructed in the 108 sub-basins of watershed. For Area, the "Ideal" fitness function value is the lowest initial capital cost initiative, which corresponds to "business as usual" scenario of no new wetlands. A stakeholder's preferences are defined by values of $w$ and $\gamma$, which define their preference for local over watershed benefits and environmental benefit over financial gain, respectively. Each preference variable can take any value between 0 and 1 , and is assigned randomly for each stakeholder from a uniform distribution. The study by Reimer et al [21] indicated that there may be some correlation between the values of $\mathrm{w}$ and $\gamma$, but as the nature of this interdependence is not specified in their study, this research will assume that $\mathrm{w}$ and $\gamma$ are independent. As and when better quantitative data on actual stakeholder conservation behavior becomes available, the relationship between $\mathrm{w}$ and $\gamma$ could be determined in a fuzzy or deterministic fashion, as appropriate.

WRESTORE uses a Likert scale of 1 to 3 to quantify user preference [14]. In order to convert the score calculated by Equation 7 to a Likert scale rating, the following relations were defined: 
- Score $_{i j}<1 / 3$ corresponds to a Likert scale user rating of 1 (i.e., "I don't like the wetland plan")

- Score $_{i j}>2 / 3$ corresponds to a Likert scale user rating of 3 (i.e., "I like the wetland plan")

- Any other value of Score $_{i j}$ corresponds to a Likert scale user rating of 2 (i.e., "I think the wetland plan is average")

This research aims to identify design alternatives that are robust to uncertainties in different degrees of stakeholder cooperation, specifically in their adoption of sub-basin scale wetland decisions in the proposed wetland plans. In one scenario of cooperation, all stakeholders could participate in adopting and allowing wetlands to be built on their property based on their Likert scale rating. Alternatively, all stakeholders could oppose the management plan, and refuse to construct wetlands on their land. Varying intermediate degrees of stakeholder participation are possible, and the distribution of participants and opponents within the watershed can also vary across the 108 sub-basins in ECW. To simulate the uncertainty in stakeholder participation in a Monte Carlo fashion, each simulated stakeholder in the watershed's sub-basin $j$ is randomly designated as a participant or opponent of the plan. If they are designated an opponent of the plan, the simulated stakeholder will automatically award any design alternative that requires them to build a wetland on their property a Score $_{i j}$ of 1 , in spite of their original preference score. Nine different extents of user participation rate were modelled, in which each rate indicates the percent of 108 sub-basins in ECW where stakeholders would be supporters of the wetland plan. The nine scenarios included:

1. Participation rate $=0 \%$. Complete opposition from the stakeholders.

2. Participation rate $=12.5 \%$. Almost complete opposition from stakeholders.

3. Participation rate $=25 \%$. Opposition from stakeholders.

4. Participation rate $=37.5 \%$. Weak opposition from stakeholders.

5. Participation rate $=50 \%$. Equal opposition and support from stakeholders.

6. Participation rate $=62.5 \%$. Weak support from stakeholders.

7. Participation rate $=75 \%$. Support from stakeholders.

8. Participation rate $=87.5 \%$. Almost complete support from stakeholders.

9. Participation rate $=100 \%$. Complete support from stakeholders.

To address the many different possible spatial distributions of location of supporters and opponents in a watershed, 100 different realizations of distribution of supporters and opponents were created for scenarios \#2 to \#8. Thus, for each single design alternative $i$ generated by the optimization algorithm, 702 realizations of $S_{c o r e} i j$ were calculated for each stakeholder $j$ based on whether they were designated as participant or opponent in a realization.

3.3 Proposed Approach for Combining Individual Stakeholder Scores to Determine Community Consensus

Work by Babbar-Sebens et al. [13] included a democratic voting procedure for determining the overall ranking of a design alternative that had been ranked by several stakeholders. In their work, the most often rating became the overall rating for the design alternative. This system successfully integrated the opinions of several users into a single rating, but it also failed to fully represent the varied opinions of individuals who did not agree with the majority rating. Consider two hypothetical design alternatives being evaluated by 9 people. The first alternative is rated as a 3 by four people, and as a 1 by five people, while the second receives five 3 ratings and four 1 ratings. According to the democratic user, the first design receives an overall rating of 1 , and the second receives an overall rating of 3 , indicating that the first design is liked by the group, while the second design is disliked. The truth, however, is that each alternative is highly controversial, with nearly equal opposition and preference, which the democratic user does not represent.

To remedy this issue, this study instead uses the number of user rating 3 awarded to each design alternative to represent the consensus opinion. This involves first calculating the number of user rating 3 received for $i^{\text {th }}$ design alternative across 702 realizations of participation scenarios and across 108 sub-basins (where the 108 stakeholders reside). This number is recorded as Counthrees $=\left\{C 3_{1}, C 3_{2} \ldots C 3_{n}\right\}$ for a set of $n$ design alternatives, where $C 3_{i}$ is the number of user rating 3 that an alternative $i$ received. Then a set $P=\left\{V_{1}, V_{2} \ldots V_{n}\right\}$ for a set of $n$ design alternatives is assessed, where $V_{i}$ is the ranking corresponding to the $i^{\text {th }}$ alternative's $C 3_{i}$ value in CountThrees. The design alternative that receives the most Likert scale rating 3 across all users and participation realizations is designated as the most preferred alternative, while the design that receives the least number of rating 3 is designated the least preferred alternatives. All remaining designs are ranked intermediately according to the number of user rating 3 they received. This approach offers two advantages over the method employed by Babbar-Sebens et al. [13]. First, this measure more accurately represents the extent of user agreement by quantifying the extent of user preference for a specific design. Second, the measure offers a higher-resolution picture of user preferences.

The Count Threes method, however, does not account for the extent of disagreement among users. Herrera-Viedma et al. [22], in their research on multiperson decision making problems (MPDM), developed a consensus model based upon two criteria: consensus and proximity. In their research, consensus measures the extent of agreement on the rating of a specific alternative, and proximity measures the distance of an individual user's rating to the consensus rating. This research adapts their measure of consensus. We begin by first defining $m$ sets of $P_{j}=$ $\left\{V_{1}^{j}, V_{2}^{j} \ldots, V_{i}^{j}, \ldots V_{n}^{j}\right\}$ for each $j^{\text {th }}$ stakeholder (where $j=1$ to $m$ and $m=108$ in this study) containing ranking of each alternative $i$ (where $i=1$ to $n$ ) assigned by the $j^{\text {th }}$ stakeholder. This set $P_{j}$ is similar to the set $P$ but involves user ratings of only $j^{\text {th }}$ stakeholder. The proximity of each stakeholder's ranking to the community ranking, for every alternative $i$, is calculated using Equation 2.

$$
\boldsymbol{p}_{j}(\boldsymbol{i})=\left(\begin{array}{ll}
\left|\begin{array}{ll}
v_{i}^{j} & v_{i}
\end{array}\right| \\
\hline n & 1
\end{array}\right)
$$

Then, community consensus for the design alternative $i$ is calculated using Equation 3.

$$
C(i)=1-\sum_{j=1}^{m} \frac{p_{j}(i)}{m}
$$


Finally, $C 3_{i}$ and $C(i)$ are treated as maximization objective function values of $i^{\text {th }}$ design alternative generated every iteration of the Interactive Optimization, and used to find the front of solutions with high community rating (estimated within CountThrees) and high community consensus (estimated by $C(i))$. These were used in addition to the watershed-scale peak flow reduction $\left(P F R_{i t}\right)$ and wetland area $\left(\right.$ Area $\left._{i t}\right)$ objective functions in the interactive, multi-objective optimization algorithm.

\section{EXPERIMENTAL RESULTS: COMPARISON OF STAKEHOLDER CONSENSUS OF INTERACTIVELY VERSUS NON- INTERACTIVELY OPTIMIZED SOLUTIONS}

Previous discussion [13] concerning incorporating stakeholders into the WRESTORE process have focused on the idea of a "tradeoff": solutions generated using stakeholderguided optimization would have reduced performance (suboptimal fitness function values) compared to the purely algorithmically optimized solutions because of the tradeoff that arises from intangible and unquantifiable fitness criteria used by the stakeholders. This research study's results are consistent with these previous findings, though with an interesting distinction. Figure 1 plots the Pareto front of the two different sets of optimized solutions and shows an interesting phenomenon. The interactive solutions occur in two bands, one of which strictly dominates the other. The two bands correspond to two different ranges of percent value of the $C 3_{i}$ objective functions value: red solutions are those whose percent value of $C 3_{i} /\left(C 3_{1}+\ldots+C 3_{n}\right)$ was found to be $26 \%$ or less, and black solutions are those whose percent values of $C 3_{i} /\left(C 3_{1}+\ldots+C 3_{n}\right)$ was found to be over $26 \%$. This result suggests that solutions that are more universally popular (have a higher percentage of number of user ratings 3 ) perform better than those that have a lower popularity (a lower percentage of number of user ratings 3). However, the non-interactive solutions (i.e., Baseline solutions in the graph) that were generated using only the

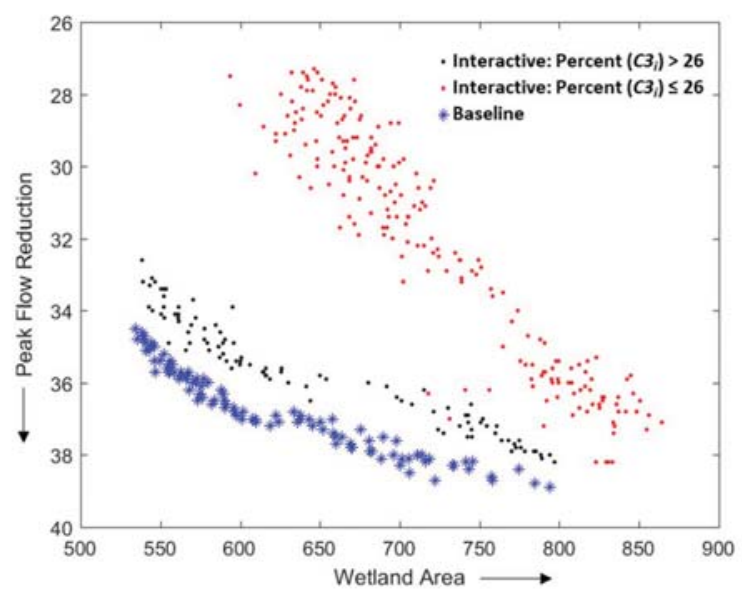

Figure 1: Pareto Fronts for Wetland Area (hectares) versus Peak Flow Reductions $\left(\mathrm{m}^{3} / \mathrm{s}\right)$ Objective Functions for Interactive (red + black solutions) and NonInteractive Optimization (blue solutions)

watershed-scale peak flow reduction and wetland area objective functions strictly dominate both fronts of interactive solutions. This dimorphic behavior may indicate that the process of convergence was not yet complete for the interactive optimization run, or that there are two distinct types of solutions that users preferred roughly interchangeably. If this is the case, then it is entirely possible that a fully convergent set of interactively optimized solutions may strictly dominate the noninteractive set of solutions, suggesting that the users guide the optimization process into a portion of the decision space not usually explored by purely algorithmic optimization.

Figure 2 is a Pareto plot with user consensus and percent of three ratings, which is a more appropriate plot for examining the community perceptions of solutions. There is only a single front of solutions from the interactive optimizations, which encompass a broad range of percent of $C 3_{i}$ values and community consensus $C(i)$ values than those of the noninteractive (Baseline) solutions. Interestingly, even though the Baseline solutions outperform interactive solutions in peak flow reduction and wetland area space (Figure 1), the community consensus of interactive solutions is better (i.e., higher) than baseline solutions when they have the same community rating (Figure 2). Further, the interactive optimization is also able to identify solutions with higher community consensus (where values of C(i) are higher than 0.7 ) even if the overall community rating is low (i.e., percent of $C 3_{i}$ is lower than 26\%). This indicates the effectiveness of interactive optimization in identifying even those solutions where there is high agreement on a low preference for the solution.

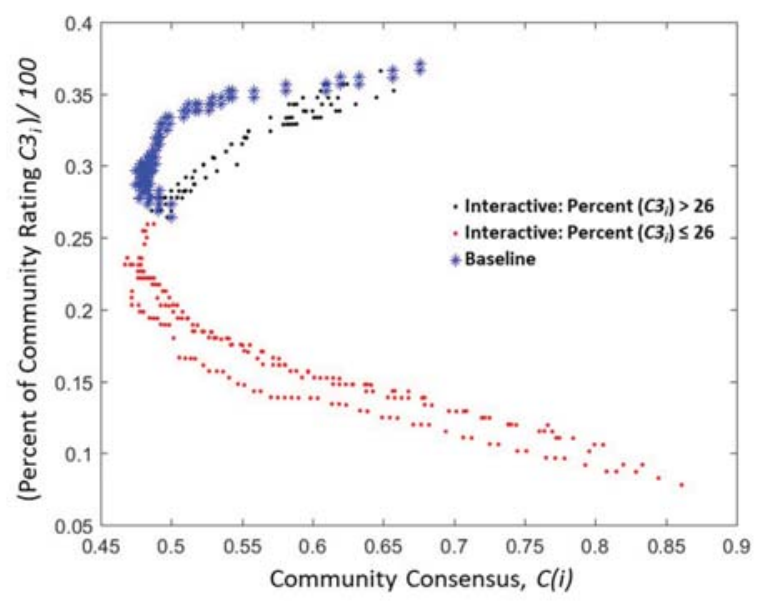

Figure 2: Community Consensus versus Community Rating for Interactive and Non-Interactive Optimization.

\section{COMMENTS AND CONCLUSIONS}

The complicated nature of watershed management plan optimization requires the continual development of novel methods to incorporate stakeholders into the design process. Stakeholder investment in the process and the resulting management plan is essential, and is strongly tied to how included in the process these stakeholders are and feel. Work completed by previous researchers associated with the WRESTORE project and other similar efforts have explored ways to incorporate decision makers and stakeholders into the 
optimization process for designing watershed management plans, and this research continues their work. This research has added two new mathematical frameworks for expressing stakeholder preferences - community consensus and community rating - in presence of stakeholder uncertainty associated with their willingness to participate in a conservation plan. While this research did not explicitly include actual stakeholder input, the method to simulate stakeholders was based upon basic information available concerning typical agricultural stakeholder opinions and preferences. When compared to the non-interactive optimization solutions that did not incorporate user opinions into the optimization process, the method proposed by this article produced solutions that did not perform as well as the non-interactive solutions with respect to minimizing wetland area and maximizing peak flow reduction. This is expected since the non-interactive solutions, unlike their interactive counterparts, do not trade off these quantitative criteria against consensus among multiple stakeholders. It is recommended that similar experiments are repeated for other watershed communities as well as other sources of uncertainties in stakeholder behaviors beyond the stochastic participation rate explore in this work. Finally, this research, in the future, may sought to assess how stakeholder-preferred wetland solutions designed considering only current climate conditions would fare when exposed to the extreme conditions of the projected future climate stresses.

\section{ACKNOWLEDGMENT}

This study has been supported by U.S. National Science Foundation (Award IDs 1332385 and 1014693) and National Oceanic and Atmospheric Administration (Award ID NA14OAR4310253).

\section{REFERENCES}

[1] S. Solomon, Water: The epic struggle for wealth, power, and civilization. Harper Perennial, 2011.

[2] A. Voinov and F. Bousquet, "Modelling with stakeholders," Environmental Modelling and Software, vol. 25, no. 11, pp. 1268 1281, 2010 .

[3] M. S. Reed, "Stakeholder participation for environmental management: A literature review," Biological Conservation, vol. 141.pp. 2417 2431, 2008 .

[4] M. L. Fisher, "Interactive optimization," Annals of Operations Research, vol. 5, no. 3, pp. 539 556, Oct. 1985.

[5] G. Klau, N. Lesh, J. Marks and M. Mitzenmacher, "Human-guided search," Journal of Heuristics, vol. 16, no. 3, pp. 289 310, 2010

[6] S. Chien, G. Rabideau, J. Willis and T. Mann, "Automating planning and scheduling of shuttle payload operations," Artificial Intelligence, vol. 114 , no. 1 2, pp. 239 255, Oct. 1999.

[7] C. D. J. Waters, "Interactive Vehicle Routeing," J Oper Res Soc, vol. 35, no. 9, pp. 821 826, Sep. 1984.

[8] H. Takagi, "Interactive evolutionary computation: fusion of the capabilities of EC optimization and human evaluation," Proceedings of the IEEE, vol. 89, no. 9, pp. 1275 1296, 2001.

[9] H. D. Nascimento and P. Eades, "User Hints for Directed Graph
Drawing," in Graph Drawing SE - 17, vol. 2265, P. Mutzel, M. Jünger, and S. Leipert, Eds. Springer Berlin Heidelberg, 2002, pp. 205219.

[10] M. Babbar-Sebens and B. Minsker, "Standard interactive genetic algorithm - Comprehensive optimization framework for groundwater monitoring design," Journal of Water Resources Planning and Management, vol. 134, no. 6, 2008

[11] M. Babbar-Sebens and B. Minsker, "A Case-Based Micro Interactive Genetic Algorithm (CBMIGA) for interactive learning and search: Methodology and application to groundwater monitoring design," Environmental Modelling and Software, vol. 25, pp. 1176 1187, 2010.

[12] M. Babbar-Sebens and B. S. Minsker, "Interactive Genetic Algorithm with Mixed Initiative Interaction for multi-criteria ground water monitoring design," Applied Soft Computing Journal, vol. 12, no. 1, 2012.

[13] M. Babbar-Sebens, S. Mukhopadhyay, V. B. Singh and A. D. Piemonti, "A web-based software tool for participatory optimization of conservation practices in watersheds," Environmental Modelling \& Software, vol. 69, pp. 111 127, Jul. 2015.

[14] A. D. Piemonti, M. Babbar-Sebens, S. Mukhopadhyay and A Kleinberg, "Interactive genetic algorithm for user-centered design of distributed conservation practices in a watershed: An examination of user preferences in objective space and user behavior," Water Resources Research, vol. 53, no. 5, 2017.

[15] Y. Y. Haimes and W. A. Hall, "Multiobjectives in water resource systems analysis: The Surrogate Worth Trade Off Method," Water Resources Research, vol. 10, no. 4, pp. 615 624, Aug. 1974.

[16] A. D. Piemonti, K. L. Macuga and M. Babbar-Sebens, "Usability evaluation of an interactive decision support system for user-guided design of scenarios of watershed conservation practices," Journal of Hydroinformatics, vol. 19, no. 5, 2017.

[17] V. B. Singh, S. Mukhopadhyay and M. Babbar-Sebens, "Decentralized Pursuit Learning Automata in Batch Mode," in Proceedings of the 6th International Conference on Soft Computing and Intelligent Systems, The 13th International Symposium on Advanced Intelligent Systems, 2012.

[18] G. A. Mendoza and H. Martins, "Multi-criteria decision analysis in natural resource management: A critical review of methods and new modelling paradigms," Forest Ecology and Management, vol. 230, no. 1 3. pp. $122,2006$.

[19] J. H. Holland, Adaptation in natural and artificial systems : an introductory analysis with applications to biology, control, and artificial intelligence. 1975.

[20] M. Babbar-Sebens, R. C. Barr, L. P. Tedesco and M. Anderson, "Spatial identification and optimization of upland wetlands in agricultural watersheds," Ecological Engineering, vol. 52, 2013.

[21] A. P. Reimer, A. W. Thompson and L. S. Prokopy, "The multidimensional nature of environmental attitudes among farmers in Indiana: Implications for conservation adoption," Agriculture and Human Values, 2012.

[22] E. Herrera-Viedma, F. Herrera and F. Chiclana, "A consensus model for multiperson decision making with different preference structures," IEEE Transactions on Systems, Man, and Cybernetics Part A:Systems and Humans., 2002. 